\title{
Reactor Antineutrinos Signal all over the world
}

\section{B. Ricci*, F.Mantovani, M. Baldoncini}

Dipartimento di Fisica e Scienze della Terra, Università and INFN, Ferrara, I-44122 Italy

E-mail: ricci@fe.infn.it

\section{J. Esposito}

INFN, Laboratori Nazionali di Legnaro, Padova I-35020- Italy

E-mail: espositodnl.infn.it

\section{Ludhova}

Dipartimento di Fisica, Università and INFN, Milano I-20133, Italy

E-mail: Iudhova@gmail.com

\section{S. Zavatarelli}

Dipartimento di Fisica, Università and INFN, Genova I-16146, Italy

E-mail: zavatarelliege.infn.it

We present an updated estimate of reactor antineutrino signal all over the world, with particular attention to the sites proposed for existing and future geo-neutrino experiment. In our calculation we take into account the most updated data on Thermal Power for each nuclear plant, on reactor antineutrino spectra and on three neutrino oscillation mechanism.

XV Workshop on Neutrino Telescopes,

11-15 March 2013

Venice, Italy

\footnotetext{
* Speaker.
} 


\begin{tabular}{|l|c|c|c|c|}
\hline Sites & $\mathrm{R}[\mathrm{TNU}]$ & $\mathrm{R}_{G}[\mathrm{TNU}]$ & $\mathrm{G}[\mathrm{TNU}]$ & $\mathrm{R}_{G} / \mathrm{G}$ \\
\hline LNGS & $85.8 \pm 4.6$ & $22.8 \pm 1.1$ & $40.3_{-5.8}^{+7.3}$ & 0.6 \\
\hline KAMIOKA & $70.1 \pm 3.7$ & $18.7 \pm 1.1$ & $31.5_{-4.1}^{+4.9}$ & 0.6 \\
\hline SUDBURY & $174.6 \pm 9.0$ & $43.1 \pm 2.1$ & $45.4_{-6.3}^{+7.5}$ & 0.9 \\
\hline PHYASALMI & $69.2 \pm 3.7$ & $17.5 \pm 0.8$ & $45.3_{-5.9}^{+7.0}$ & 0.4 \\
\hline FREJUS & $587.9 \pm 31.0$ & $134.0 \pm 7.1$ & $42.4_{-6.2}^{+7.6}$ & 3.2 \\
\hline HOMESTAKE & $27.7 \pm 1.5$ & $7.3 \pm 0.3$ & $48.7_{-6.9}^{+8.4}$ & 0.1 \\
\hline HAWAII & $3.4 \pm 0.2$ & $0.9 \pm 0.04$ & $12.0_{-0.6}^{+0.7}$ & 0.1 \\
\hline CURACAO & $9.5 \pm 0.5$ & $2.5 \pm 0.1$ & $29.3_{-3.3}^{+4.2}$ & 0.1 \\
\hline
\end{tabular}

Table 1: Comparison between expected reactor $(\mathrm{R})$ and geo $(\mathrm{G})$ antineutrino signal. $\mathrm{R}_{G}$ indicates the reactor signale expected in the geo neutrino energy window $\left(E_{\bar{v}}<3.26 \mathrm{MeV}\right)$. Geo-neutrino signal has been calculated following the approach described in [14]. $1 \mathrm{TNU}=1 \mathrm{event} / \mathrm{year} / 10^{32}$ protons.

\section{Discussion}

Antineutrinos from the decay chains of ${ }^{238} \mathrm{U}$ and ${ }^{232} \mathrm{Th}$ existing in the Earth interior (the so called geo-neutrinos) have been recently detected both by Kamland [1] and by Borexino [2] experiments. Future experiments for geo-neutrinos detection have been proposed (or starting) in several location in the world (e.g. SNO+ in Canada [3], Lena project in Europe [他 and Hawaii Anti-Neutrino Observatory[5]).

The main source of background of such experiments is given by antineutrino produced by nuclear plants. These particles account for a signal almost always larger than geo-neutrinos one, see Table 1. So a detailed calculation of reactor antineutrino flux in mandatory for an accurate measurements of geo-neutrinos.

With this aim, we performed a calculation of reactor antineutrinos flux all over the world. Previus analysis has been presented, for instance, in ref. [6] and [7]. Now we will show an updated estimate of reactor antineutrino signal, with particular attention to the sites proposed for the new geo-neutrino experiments. In our calculation we take into account the most updated data on Thermal Power for each nuclear plant, on reactor antineutrino spectra and on three neutrino oscillation mechanism. The expected reactor antineutrino signal has been calculated as follows:

$$
N_{e v}=\varepsilon N_{p} \tau \sum_{r=1}^{N_{\text {react }}} \frac{P_{r}}{4 \pi L_{r}^{2}}<L F_{r}>\int d E_{\bar{v}_{e}} \sum_{i=1}^{4} \frac{f_{i}}{E_{i}} \phi_{i}\left(E_{\bar{v}_{e}}\right) \sigma\left(E_{\bar{v}_{e}}\right) P_{e e}\left(E_{\bar{v}_{e}} ; \hat{\theta}, L_{r}\right)
$$

where $\varepsilon$ is detector efficiency, $N_{p}$ is the number of target proton, $\tau$ is period of data taking, index $r$ cycles over the $N$ reactors considered, $L_{r}, P_{r}$ and $\left\langle L F_{r}\right\rangle$ are the distance, the nominal thermal power and the averaged Load Factor of reactor $r$, respectively. The index $i$ stands for the $\mathrm{i}$-th spectral component in the set ${ }^{235} \mathrm{U},{ }^{238} \mathrm{U},{ }^{239} \mathrm{Pu}$, and $\left.{ }^{241} \mathrm{Pu}\right), f_{i}$ is the power fraction of the component $i$, as reported in[2], $E_{i}$ is the average antineutrino energy per fission of the component $i$ [1]], $\phi\left(E_{\bar{v}}\right)$ is the anti-neutrino flux per fission of the $i^{\text {th }}$ component, as recently calculated in ref.[9], $\sigma\left(E_{\bar{v}}\right)$ is the inverse beta decay cross section[12] and $P_{e e}$ is the survival probability of the reactor antineutrinos of energy $E_{\bar{v}}$ traveling the baseline $L_{r}$, depending on the mixing parameters $\hat{\theta}$.

In Eq. (1.1) we assume a $100 \%$ detection efficiency, for a detector containing $10^{32}$ target protons and operating continuously for 1 year. In particular we consider the nuclear cores all over 


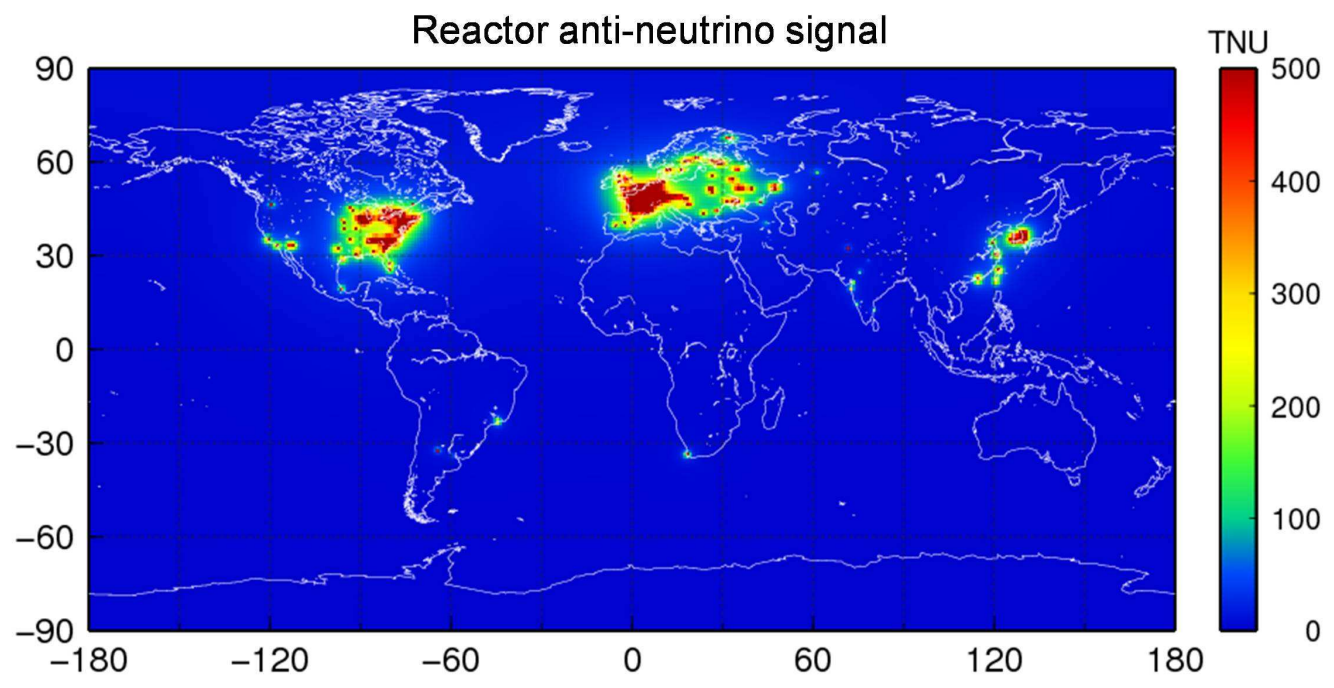

Figure 1: A worldwide map of reactor antineutrinos signal. $1 \mathrm{TNU}=1$ events/yr/ $10^{32}$ target protons

the world, operating in the year 2012. Information on the nominal thermal power and monthly load factor for each nuclear cores originates from International Agency of Atomic Energy (IAEA) [8]. Concerning survival probability, we assumed a three flavour vacuum oscillation mechanism with $P_{e e}$ as in in ref.[10], and mixing parameters from ref.[13].

The results of our calculation are reported in Table1 and in Fig.1. We also performed a analysis on the sources of uncertainty in reactor signal prediction, see for details. The total uncertainty is of the order of $5 \%$, the main contributions (i.e. greater than $2 \%$ ) arising from $\theta_{12}$ mixing angle, antineutrino spectrum, fuel composition and thermal power.

One can see that, due to reactors shutdown occurred in 2012, Kamioka became a suitable site for detecting geo-neutrinos, comparable to LNGS. A new European geo-neutrino detector located at Frejus Laboratory requires a detailed knowledge of closeby reactors; the choice of Phyasalmi looks better in this respect. Of course Hawaii and Curacao are wonderful places for geo-neutrino studies due to their position far away from any nuclear plants of the world. The same holds for Homestake. In the near future, the $\mathrm{SNO}+$ experiment, with a quite reasonable ratio $\mathrm{R}_{G} / \mathrm{G}$, will provide more information about Earth's interior.

\section{References}

[1] T. Araki et al. (KamLAND Collaboration), Nature 436 (2005) 499 ; S. Abe et al. (KamLAND Collaboration, Phys. Rev. Lett. 100 (2008) 221803; A. Gando et al. (KamLAND Collaboration), Nature Geoscience 4 (2011) 647; A. Gando et al. (KamLAND Collaboration), arXive:1303.4667.

[2] G. Bellini et al. (Borexino Collaboration), Phys. Lett. B 687 (2010) 299; G. Bellini et al. (Borexino Collaboration), Phys. Lett. B 722 (2013) 295.

[3] SNO+ Collaboration: C. Kraus et al. Prog. Part. Nucl. Phys. 57 (2006) 150; M. Chen et al., 2008, arXiv:0810.3694; V. Lozza et al., 2012, arXiv:1201.6599.

[4] M. Wurm et al. Astrop.Phys. 35 (2012) 685. 
[5] S. T. Dye et al., Earth Moon Planets 99 (2006) 241; J. G. Learned, S. T. Dye, and S. Pakvasa, 2008, arXiv:0810.4975.

[6] G. Fiorentini, M.Lissia and F. Mantovani, Phys. Rep. 453 (2007) 117.

[7] B.Ricci et al, talk at Neutrino Geoscience 2010, LNGS, 6-8 October 2010. http://geoscience.lngs.infn.it/

[8] J. Mandula, Nuclear Power Engeneering Section, IAEA, Vienna, private comunication 2013.

[9] Th. A. Mueller et al., Phys. Rev. C 83 (2011) 054615.

[10] G. Fiorentini et al., Phys. Rev. D 86 (2012) 033004.

[11] P. Huber and T. Schwetz, Phys. Rev. D 70 (2004) 053011.

[12] A. Strumia and F. Vissani, Phys. Lett. B 564 (2003) 42.

[13] G. Fogli et al., Phys. Rev. D 86 (2012) 033004.

[14] Y. Huang et al., submitted to Geochem. Geophys. Geosyst., arXiv:1301.0365 [physics.geo-ph]. 